\title{
Control Technique of Maximum Local Lyapunov Exponent on Stable Periodic Solution in Continuous-Time Non-Autonomous Dynamical Systems
}

\author{
Ken'ichi Fujimoto, Tomohiro Otsu, Hiroyuki Kitajima, and Tetsushi Ueta
}

\begin{abstract}
We previously proposed a parametric controller to avoid undesirable bifurcations of stable fixed and periodic points in discrete-time dynamical systems. The parameter regulation is derived from an optimization problem on the maximum local Lyapunov exponent and a method of steepest descent. In this paper, on the basis of the ideas and a stroboscopic mapping that transforms the trajectory of a continuous-time periodic solution into a sequence of points, we propose a technique to control the maximum local Lyapunov exponent on stable periodic oscillations in continuous-time non-autonomous dynamical systems.
\end{abstract}

Keywords-Maximum local Lyapunov exponent, parametric control, stroboscopic mapping, stable periodic oscillation, continuous-time non-autonomous dynamical system.

\section{INTRODUCTION}

Nonlinear dynamical systems expressed by parameterized difference or differential equations are widely used for mathematical modeling of physical systems in many fields [1], [2]. In general, the values of system parameters are set so that a desired behavior can appear in a steady state. However, a dynamical system may not work as expected for any reason, e.g., disappearance of the desired behavior for occurrence of bifurcation [3], [4].

For stable fixed and periodic points in discrete-time dynamical systems, we previously proposed a novel parametric controller to avoid undesirable bifurcation of stable ones [5]-[7]. This is one of bifurcation control techniques [8], and the parameter regulation is derived from an optimization problem on the maximum local Lyapunov exponent [9]-[12] and a method of steepest descent. The effectiveness of the proposed method has been demonstrated through numerical experiments for stable fixed or periodic points observed in Hènon map [13] and Kawakami map [14].

The proposed control technique, however, cannot be directly applied to stable periodic solutions in continuous-time dynamical systems. To avoid undesirable bifurcation of continuous-time solutions, we need a new scheme of parametric control methodology. In this paper, on the basis of the

Manuscript received May 20, 2016. This work was supported by JSPS KAKENHI Grant Number 16K00332.

K. Fujimoto is with Kagawa University, Kagawa 760-8521, Japan

T. Otsu was with Tokushima University, Tokushima 770-8506, Japan

H. Kitajima is with Kagawa University, Kagawa 761-0396, Japan

T. Ueta is with Tokushima University, Tokushima 770-8506, Japan aforementioned ideas and a stroboscopic mapping (otherwise known as Poincarè map) that transforms the trajectory of a continuous-time solution into a sequence of points, we propose a technique to control the maximum local Lyapunov exponent on stable periodic oscillations in continuous-time non-autonomous dynamical systems.

\section{PRELIMINARY}

\section{A. Periodic Solution and its Stability}

Let us consider a continuous-time non-autonomous dynamical system defined by

$$
\frac{d \boldsymbol{x}}{d t}=\boldsymbol{f}(t, \boldsymbol{x}, \boldsymbol{p})
$$

where $t \in \boldsymbol{R}$ denotes continuous time, $\boldsymbol{x}=\left(x_{1}, x_{2}, \ldots, x_{N}\right)^{\mathrm{T}} \in \boldsymbol{R}^{N}$ is the vector of state variables, $\boldsymbol{p}=\left(p_{1}, p_{2}, \ldots, p_{M}\right)^{\mathrm{T}} \in \boldsymbol{R}^{M}$ is the vector of system parameters, and the superscript symbol $\mathrm{T}$ represents the transpose of a vector. We now assume that $\boldsymbol{f}$ is periodical on time, and its period is $\Psi$, i.e.,

$f(t+\Psi, x, p)=f(t, x, p)$

is satisfied.

We express a solution to (1) as

$\boldsymbol{x}(t)=\varphi\left(t, x^{(0)}, p\right)$,

where $\boldsymbol{x}^{(0)}=\boldsymbol{x}(0) \in R^{N}$ represents an initial values at $t=0$, then a periodic solution with period $L \Psi(L=1,2, \ldots)$ can be defined by

$\varphi\left(t+L \Psi, x^{(0)}, p\right)=\varphi\left(t, x^{(0)}, p\right)$

Note that a solution with $L=1$ is called fundamental harmonic solution, otherwise, sub-harmonic solution of order $L$.

We now assume that local sections $\Omega:=\left\{x \in R^{N} \mid t=k \Psi, k=0,1\right.$, $2, \ldots\}$ are arranged in the state space in (1). To map a point of a solution's trajectory, $\boldsymbol{x}^{(k)}=\boldsymbol{x}(t)$ at $t=k \Psi$, on the local section at the time to the next point $\boldsymbol{x}^{(k+1)}$ in the next local section at $t=(k+1) \Psi$, we define a stroboscopic mapping $\boldsymbol{S}$ as 


$$
S: R^{N} \rightarrow R^{N} ; x^{(k)} \mapsto x^{(k+1)}=\varphi\left((k+1) \Psi, x^{(k)}, p\right)
$$

To simplify derivation of a parametric controller in the followings, we assume that $\boldsymbol{S}$ is differentiable with respect to $t$, $\boldsymbol{x}$, and $\boldsymbol{p}$ as many times as needed.

This mapping enables us to express the trajectory of a continuous-time solution to (1) as a sequence of points on local sections. For example, a point $\left(\boldsymbol{x}^{*}\right)$ on a fundamental harmonic solution maps to the same point on all local section, i.e., $\boldsymbol{S}\left(\boldsymbol{x}^{*}\right)-\boldsymbol{x}^{*}=\mathbf{0}$. It is, therefore, called as fixed point. Likewise, since a point $\left(\boldsymbol{x}^{*}\right)$ on a sub-harmonic solution of order $L$ satisfies $\boldsymbol{S}^{L}\left(\boldsymbol{x}^{*}\right)-\boldsymbol{x}^{*}=\mathbf{0}$, we call it $L$-periodic point. Thus, by using the mapping, we can handle a periodic solution to (1) like a fixed or periodic point in a discrete-time dynamical system.

The stability of a fixed or periodic point $\left(\boldsymbol{x}^{*}\right)$ can be discussed by eigenvalues $\mu_{i} \in \boldsymbol{C}(i=1,2, \ldots, N)$ of the Jacobian matrix derived from $\boldsymbol{S}\left(\boldsymbol{x}^{*}\right)-\boldsymbol{x}^{*}=\mathbf{0}$ or $\boldsymbol{S}^{L}\left(\boldsymbol{x}^{*}\right)-\boldsymbol{x}^{*}=\mathbf{0}$, respectively. The fixed or periodic point $\left(x^{*}\right)$ is stable only if $\left|\mu_{i}\right|<1$ for all $i$, which corresponds to the situation that all the eigenvalues are within the unit circle in the complex plane. On the other hand, when one or more eigenvalues are on the circumference of the unit circle, a stable fixed or periodic one becomes unstable and bifurcates. Note that Kawakami et al. [4] summarized types of general bifurcation, conditions that each bifurcation occurs, and a computational technique to find bifurcation points.

\section{B. Maximum Local Lyapunov Exponent}

Let us assume that the limit of the sequence $\boldsymbol{x}^{(k)}$ starting from an initial point $\boldsymbol{x}^{(0)}$ exists, besides, the limit set is a stable fixed or periodic point. We express the derivative of $\boldsymbol{x}^{(k)}$ with respect to $\boldsymbol{x}^{(0)}$ as

$$
\frac{\partial x^{(k)}}{\partial x^{(0)}}=D S^{k}\left(x^{(0)}, p\right)=\frac{\partial S^{k}\left(x^{(0)}, p\right)}{\partial x^{(0)}}=\prod_{\ell=1}^{k} D S\left(x^{(\ell)}, p\right)
$$

and define the maximum Lyapunov Exponent on $\boldsymbol{x}^{(k)}$ by

$$
\begin{aligned}
\Lambda\left(x^{(0)}, p\right) & =\lim _{\tau \rightarrow \infty} \frac{1}{\tau} \ln \frac{\left\|D S^{\tau}\left(x^{(0)}, p\right) \cdot v^{(0)}\right\|}{\left\|v^{(0)}\right\|} \\
& =\lim _{\tau \rightarrow \infty} \frac{1}{\tau} \sum_{k=0}^{\tau-1} \ln \frac{\left\|D S\left(x^{(k)}, p\right) \cdot v^{(k)}\right\|}{\left\|v^{(0)}\right\|},
\end{aligned}
$$

where $\boldsymbol{v}^{(0)}$ is a nearby point at the vicinity of $\boldsymbol{x}^{(0)}$, and $\|\cdot\|$ represents the Euclidean norm of a vector. Regardless of a given $\boldsymbol{v}^{(0)}$, the maximum Lyapunov exponent takes the same value for almost all initial points in the basin of a stable fixed or periodic point [9]. For example, a stable fixed or periodic point is present under the values of $\boldsymbol{p}$ if $\Lambda\left(\boldsymbol{x}^{(0)}, \boldsymbol{p}\right)$ takes a negative value. When the value of $\Lambda\left(x^{(0)}, p\right)$ approaches to zero, the values of $\boldsymbol{p}$ are close to a bifurcation point of the stable fixed or periodic point.

Computing the maximum Lyapunov exponent based on (7) is numerically difficult because it is defined in infinite time.
Instead of the maximum Lyapunov exponent, we introduce the maximum local Lyapunov exponent [11], [12] that is defined in finite time:

$\lambda\left(x^{(0)}, p, \tau\right)=\frac{1}{\tau} \sum_{k=0}^{\tau-1} \ln \frac{\left\|D S\left(x^{(k)}, p\right) \cdot v^{(k)}\right\|}{\left\|v^{(0)}\right\|}$.

Here, we set the discrete-time interval $\tau$ to compute (8) to $K L$ with a positive large integer $K$, which the interval corresponds to $t \in[0, K L \Psi)$, and normalize $\boldsymbol{v}^{(k)}$ so that the value of $\left\|\boldsymbol{v}^{(k)}\right\|$ always becomes one as

$v^{(k)}=w^{(k)} /\left\|\boldsymbol{w}^{(k)}\right\|$,

$\boldsymbol{w}^{(k+1)}=D \boldsymbol{S}\left(\boldsymbol{x}^{(k)}, \boldsymbol{p}\right) \cdot \boldsymbol{v}^{(k)}$,

where $\boldsymbol{w}^{(0)} \in \boldsymbol{R}^{N}$ is given as a perturbation of $\boldsymbol{x}^{(0)}$. This normalization (9a) is due to relieve the difficulty in the numerical computation of (8).

\section{Methodology of Parametric Control}

We consider the situation that bifurcation of a stable fundamental harmonic or sub-harmonic solution of order $L$ to (1), which corresponds to a stable fixed or periodic point of a stroboscopic mapping in (5), can occur by changing the value of one or more parameter values. Under the critical situation in the context that undesirable bifurcation may occur, we treat the problem to control the maximum local Lyapunov exponent so that it becomes a negative value that we prescribe in advance.

To simplify the problem, we assume that the values of $\boldsymbol{p}$ are constant for the duration of $t \in[m \tau \Psi,(m+1) \tau \Psi),(m=0,1,2, \ldots)$. From this assumption, (8), and (9), we can compute the value of $\lambda^{(m \tau)}=\lambda\left(\boldsymbol{x}^{(m \tau)}, \boldsymbol{p}^{(m \tau)}, \tau\right)$ at $t=(m+1) \tau \Psi-\Psi$, i.e., it is defined as

$\lambda^{(m \tau)}=\frac{1}{\tau} \sum_{k=m \tau}^{(m+1) \tau-1} \ln \|\boldsymbol{w}(k+1)\|$

To design a parametric controller so as not to make bifurcation for forcibly change of a system-parameter value, we consider the minimization problem of an objective function defined by

$J\left(\lambda^{(m \tau)}\right)=\frac{1}{2}\left(\lambda^{(m \tau)}-\lambda^{*}\right)^{2}$

where $\lambda^{*}$ denotes the target value of the maximum local Lyapunov exponent to be controlled and is set to a negative value.

Now, let $p$ be a controlled parameter, which corresponds to one of $\boldsymbol{p}$, and we assume that $\lambda^{(m \tau)}$ is differentiable with respect to $p$. Under the assumption, the method of steepest descent yields a gradient system of (11) with respect to $p$ defined by 
$p^{((m+1) \tau)}-p^{(m \tau)}=-\eta \frac{\partial J\left(\lambda^{(m \tau)}\right)}{\partial p}=-\eta\left(\lambda^{(m \tau)}-\lambda^{*}\right) \frac{\partial \lambda^{(m \tau)}}{\partial p}$

i.e., the parameter regulation of $p$ is defined as

$p^{((m+1) \tau)}=p^{(m \tau)}-\eta\left(\lambda^{(m \tau)}-\lambda^{*}\right) \frac{\partial \lambda^{(m \tau)}}{\partial p}$

where $\eta$ is a user-defined positive parameter. The differential value in (13) can be obtained by partially differentiating (10) with respect to $p$ as

$\frac{\partial \lambda^{(m \tau)}}{\partial p}=\frac{1}{\tau} \sum_{k=m \tau}^{(m+1) \tau-1} \frac{1}{\left\|w^{(k+1)}\right\|} \cdot \frac{\partial\left\|\boldsymbol{w}^{(k+1)}\right\|}{\partial p}$

where

$$
\frac{\partial\left\|\boldsymbol{w}^{(k+1)}\right\|}{\partial p}=\frac{\boldsymbol{w}^{(k+1)^{\mathrm{T}}}}{\left\|\boldsymbol{w}^{(k+1)}\right\|} \cdot \frac{\partial \boldsymbol{w}^{(k+1)}}{\partial p}
$$

From (9), the values of $\partial \boldsymbol{w}^{(k+1)} / \partial p$ can be calculated by

$$
\frac{\partial \boldsymbol{w}^{(k+1)}}{\partial p}=\frac{\partial D \boldsymbol{S}\left(\boldsymbol{x}^{(k)}, \boldsymbol{p}\right)}{\partial p} \cdot \boldsymbol{v}^{(k)}+D \boldsymbol{S}\left(\boldsymbol{x}^{(k)}, \boldsymbol{p}\right) \cdot \frac{\partial \boldsymbol{v}^{(k)}}{\partial p}
$$

where the initial values of $\partial w / \partial p$ and $\partial v / \partial p$ are set to zero because we arbitrarily give the initial points of $\boldsymbol{w}$ and $\boldsymbol{v}$ regardless of the value of $p$.

Calculating the right-hand side of (15) is more complex than that of the case [4]-[6] that the original target is a fixed or periodic point in discrete-time dynamical systems because the original one is a continuous-time solution to (1). The computational procedure is as follows. As described in (5) and (6), $D \boldsymbol{S}\left(\boldsymbol{x}^{(k)}, \boldsymbol{p}\right)$ on the right-hand side of (15) means the derivative of $\boldsymbol{S}(\boldsymbol{x}(k \Psi), \boldsymbol{p})=\boldsymbol{\varphi}((k+1) \Psi, \boldsymbol{x}(k \Psi), \boldsymbol{p})$ with respect to $\boldsymbol{x}(k \Psi)$ that corresponds to the initial point in the duration of $t \in[k \Psi,(k+1) \Psi]$. Therefore, the $(i, j)$ th element of the matrix is defined by

$$
D S(x(k \Psi), p)_{i j}=\frac{\partial \varphi_{i}((k+1) \Psi, x(k \Psi), p)}{\partial x_{j}(k \Psi)},
$$

or

$$
D \boldsymbol{S}\left(\boldsymbol{x}^{(k)}, \boldsymbol{p}\right)_{i, j}=\frac{\partial \varphi_{i}\left((k+1) \Psi, x^{(k)}, \boldsymbol{p}\right)}{\partial x_{j}^{(k)}} .
$$

To calculate these values, we revisit the continuous-time non-autonomous dynamical system described by (1). From (1) and (3), we have

$$
\frac{d \varphi\left(t, x^{(0)}, p\right)}{d t}=f\left(t, x^{(0)}, p\right)
$$

and its derivative with respect to $\boldsymbol{x}^{(0)}$ is obtained as

$$
\frac{\partial}{\partial x^{(0)}}\left(\frac{d \varphi\left(t, x^{(0)}, p\right)}{d t}\right)=\frac{\partial}{\partial x^{(0)}} f\left(t, \varphi\left(t, x^{(0)}, p\right), p\right)
$$

Since the order of differentiation on the left-hand side is commutative, we obtain the first variational equation of (17) with respect to initial value,

$$
\frac{d}{d t}\left(\frac{\partial \varphi\left(t, x^{(0)}, p\right)}{\partial x^{(0)}}\right)=\frac{\partial f\left(t, \varphi\left(t, x^{(0)}, p\right), p\right)}{\partial x} \cdot \frac{\partial \varphi\left(t, x^{(0)}, p\right)}{\partial x^{(0)}}
$$

and the initial point of this differential equation is as $\partial \boldsymbol{\varphi}\left(t, \boldsymbol{x}^{(0)}\right.$, $\boldsymbol{p}) / \partial \boldsymbol{x}^{(0)}=\boldsymbol{E}$, where $\boldsymbol{E}$ denotes the $N \times N$ identity matrix. In addition, $\partial \boldsymbol{f}\left(t, \boldsymbol{\varphi}\left(t, \boldsymbol{x}^{(0)}, \boldsymbol{p}\right), \boldsymbol{p}\right) / \partial \boldsymbol{x}$ is the Jacobian matrix of $\boldsymbol{f}$ in (17), and all the elements can be algebraically calculated from a given $f$ in advance. Therefore, we can calculate the value of the right-hand side of (16) by numerically integrating (19) with the initial point from $t=k \Psi$ to $t=(k+1) \Psi$.

The elements of $\partial D \boldsymbol{S}\left(\boldsymbol{x}^{(k)}, \boldsymbol{p}\right) / \partial p$ on the first term of the right-hand side of (15) can be calculated from the partial derivative of (19) with respect to $p$ as

$$
\begin{aligned}
& \frac{d}{d t}\left(\frac{\partial^{2} \varphi\left(t, x^{(0)}, p\right)}{\partial x^{(0)} \partial p}\right) \\
& =\frac{\partial f\left(t, \varphi\left(t, x^{(0)}, p\right), p\right)}{\partial x} \cdot \frac{\partial^{2} \varphi\left(t, x^{(0)}, p\right)}{\partial x^{(0)} \partial p} \\
& +\frac{\partial^{2} f\left(t, \varphi\left(t, x^{(0)}, p\right), p\right)}{\partial x^{2}} \cdot \frac{\partial \varphi\left(t, x^{(0)}, p\right)}{\partial x^{(0)}} \cdot \frac{\partial \varphi\left(t, x^{(0)}, p\right)}{\partial p} \\
& +\frac{\partial^{2} f\left(t, \varphi\left(t, x^{(0)}, p\right), p\right)}{\partial x \partial p} \cdot \frac{\partial \varphi\left(t, x^{(0)}, p\right)}{\partial x^{(0)}}
\end{aligned}
$$

with the initial point, $\partial^{2} \boldsymbol{\varphi}\left(t, \boldsymbol{x}^{(0)}, \boldsymbol{p}\right) / \partial \boldsymbol{x}^{(0)} \partial p=\mathbf{0}$ at $t=k \Psi$. In the right-hand side of (20), as aforementioned, we can algebraically calculate all the elements of $\partial \boldsymbol{f}\left(t, \boldsymbol{\varphi}\left(t, \boldsymbol{x}^{(0)}, \boldsymbol{p}\right), \boldsymbol{p}\right) / \partial \boldsymbol{x}$ from a given $\boldsymbol{f}$ in advance. Its derivatives with respect to $\boldsymbol{x}$ and $p, \partial^{2} \boldsymbol{f}(t, \boldsymbol{\varphi}(t$, $\left.\left.\boldsymbol{x}^{(0)}, \boldsymbol{p}\right), \boldsymbol{p}\right) / \partial \boldsymbol{x}^{2}$ and $\partial^{2} f\left(t, \boldsymbol{\varphi}\left(t, \boldsymbol{x}^{(0)}, \boldsymbol{p}\right), \boldsymbol{p}\right) / \partial \boldsymbol{x} \partial p$, can be also calculated algebraically. In addition, the values of $\partial \varphi\left(t, \boldsymbol{x}^{(0)}\right.$, $\boldsymbol{p}) / \partial \boldsymbol{x}^{(0)}$ have been already computed.

Like the way of derivation of (19), we partially differentiate (17) with respect to $p$ and permute the order of differentials as

$$
\begin{array}{r}
\frac{d}{d t}\left(\frac{\partial \varphi\left(t, x^{(0)}, p\right)}{\partial p}\right)=\frac{\partial f\left(t, \varphi\left(t, x^{(0)}, p\right), p\right)}{\partial x} \cdot \frac{\partial \varphi\left(t, x^{(0)}, p\right)}{\partial p} \\
+\frac{\partial f\left(t, \varphi\left(t, x^{(0)}, p\right), p\right)}{\partial p}
\end{array}
$$

and the initial point such that $\partial \boldsymbol{\varphi}\left(t, \boldsymbol{x}^{(0)}, \boldsymbol{p}\right) / \partial p=\mathbf{0}$. Here, we can algebraically calculate all the elements of $\partial f\left(t, \varphi\left(t, \boldsymbol{x}^{(0)}, \boldsymbol{p}\right), \boldsymbol{p}\right) / \partial \boldsymbol{x}$ and $\partial \boldsymbol{f}\left(t, \boldsymbol{\varphi}\left(t, \boldsymbol{x}^{(0)}, \boldsymbol{p}\right), \boldsymbol{p}\right) / \partial p$ for a given $\boldsymbol{f}$ in advance. Therefore, 
the values of $\partial \boldsymbol{\varphi}\left(t, \boldsymbol{x}^{(0)}, \boldsymbol{p}\right) / \partial p$ in (20) can be computed by numerically integrating (21) with the initial point from $t=k \Psi$ to $t=(k+1) \Psi$. Thus, the values of $\partial D \boldsymbol{S}\left(\boldsymbol{x}^{(k)}, \boldsymbol{p}\right) / \partial p$ in $(15)$ can be also computed by integrating (20) with the initial point from $t=k \Psi$ to $t=(k+1) \Psi$, numerically. Note that the four ordinary differential equations, which are (17) and (19)-(21), can be integrated in parallel.

Calculation of $\boldsymbol{v}^{(k)}$ in (15) is based on algebraic calculation described in (9) because we already had the values of $\boldsymbol{D S}\left(\boldsymbol{x}^{(k)}\right.$, $\boldsymbol{p})$. The partial derivative of $\boldsymbol{v}^{(k+1)}$ with respect to $p$ can be calculated by

$$
\frac{\partial \boldsymbol{v}^{(k+1)}}{\partial p}=\frac{1}{\left\|\boldsymbol{w}^{(k+1)}\right\|} \cdot \frac{\partial \boldsymbol{w}^{(k+1)}}{\partial p}-\frac{1}{\left\|\boldsymbol{w}^{(k+1)}\right\|^{2}} \cdot \frac{\partial\left\|\boldsymbol{w}^{(k+1)}\right\|}{\partial p} \cdot \boldsymbol{w}^{(k+1)}
$$

where the initial values of $\partial \boldsymbol{v} / \partial p$ are set to zero as aforementioned in (15). In addition, the values of $\partial w^{(k+1)} / \partial p$ and $\partial\left\|\boldsymbol{w}^{(k+1)}\right\| / \partial p$ can be assigned with the values computed from (15) and (14b), respectively.

The updating procedure of the $p$ value to control the maximum local Lyapunov exponent on a stable sub-harmonic solution of order $L$ is summarized as the following algorithm.

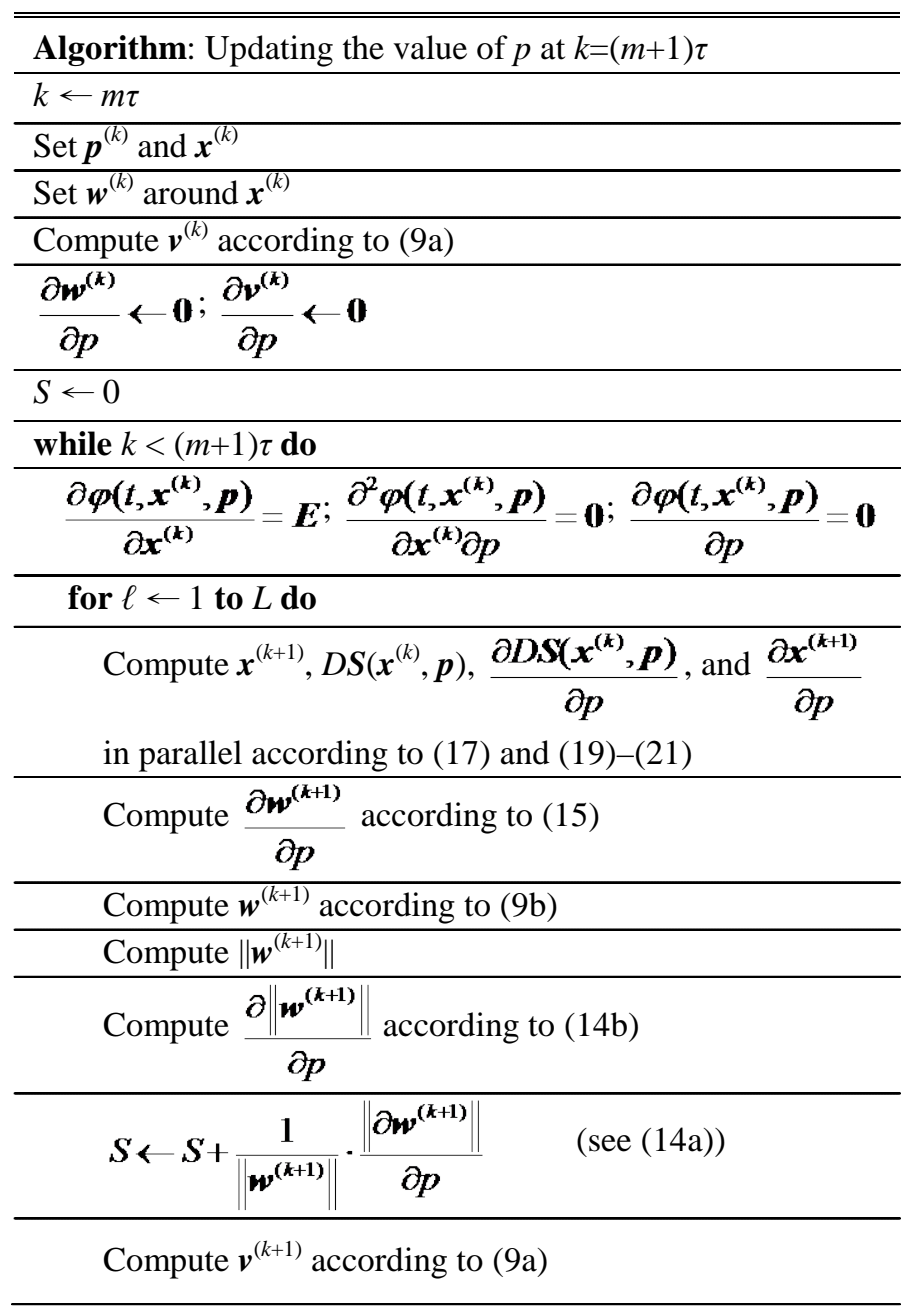

\begin{tabular}{l}
\hline Compute $\frac{\partial v^{(k+1)}}{\partial p}$ according to (22) \\
\hline$k \leftarrow k+1$ \\
\hline end for \\
\hline end while \\
$\frac{\partial \lambda^{(m \tau)}}{\partial p} \leftarrow \frac{S}{\tau} \quad$ (see (14a)) \\
\hline$p \leftarrow p^{(m \tau)}-\eta\left(\lambda^{(m \tau)}-\lambda^{*}\right) \frac{\partial \lambda^{(m \tau)}}{\partial p} \quad$ (see (13)) \\
\hline \hline
\end{tabular}

\section{CONCLUSION}

In this paper, we proposed a parametric control technique for the maximum local Lyapunov exponent on stable periodic solutions observed in continuous-time non-autonomous dynamical systems. It is expected that the proposed technique is available to avoid undesirable bifurcation of such solutions.

\section{REFERENCES}

[1] S. Wiggins, Introduction to Applied Nonlinear Dynamical Systems and Chaos, 2nd ed. New York; Springer, 2003.

[2] M. A. Jordán (Ed.), Discrete Time Systems, Rijeka; InTech, 2011. http://dx.doi.org/10.5772/599

[3] J. Sun, F. Amellal, L. Glass, and J. Billette, "Alternans and period-doubling bifurcations in atrioventricular nodal conduction," $J$. Theor. Biol., vol. 173, pp. 79-91, Mar. 1995.

http://dx.doi.org/10.1006/jtbi.1995.0045

[4] K. Tsumoto, T. Ueta, T. Yoshinaga, and H. Kawakami, "Bifurcation analyses of nonlinear dynamical systems: From theory to numerical computations," Nonlinear Theory and Its Applications, IEICE, vol. 3, pp.458-476, Oct. 2012. http://dx.doi.org/10.1587/nolta.3.458

[5] K. Fujimoto, T. Otsu, T. Yoshinaga, T. Ueta, H. Kitajima, and K. Aihara, "Controlling method to avoid bifurcations of periodic points using maximum Lyapunov exponent,” Proc. 2013 Int. Symp. on Nonlinear Theory and its Applications, 2013, pp. 158-161.

[6] K. Fujimoto and K. Aihara, "Bifurcation avoidance control of stable periodic points using the maximum local Lyapunov exponent," Nonlinear Theory and Its Applications, IEICE, vol. 6, pp. 2-14, Jan. 2015.

http://dx.doi.org/10.1587/nolta.6.2

[7] K. Fujimoto, T. Yoshinaga, T. Ueta, K. Aihara, "Parametric Control to Avoid Bifurcation Based on Maximum Local Lyapunov Exponent," in Analysis and Control of Complex Dynamical Systems, K. Aihara, J. Imura, and T. Ueta, Eds. Springer, 2015, ch. 5, pp. 49-55.

[8] G. Chen, J. L. Moiola, and H. O. Wang, "Bifurcation control: theories, methods, and applications," Int. J. Bifurcation Chaos, vol. 10, pp.511-548, Mar. 2000. http://dx.doi.org/10.1016/S0218-1274(00)00036-0

[9] V. I. Oseledec, "A multiplicative ergodic theorem: Lyapunov characteristic numbers for dynamical systems," Trans. Moscow Math. Soc., vol. 19, pp. 197-231, Jan. 1968.

[10] A. Wolf, J. B. Swift, H. L. Swinney, and J. A. Vastano, "Determining Lyapunov exponents from a time series," Physica D, vol. 16, pp. 285-317, July 1985.

http://dx.doi.org/10.1016/0167-2789(85)90011-9

[11] H. D. I. Abarbanel, R. Brown, and M. B. Kennel, "Local Lyapunov exponents computed from observed data," J. Nonlinear Sci., vol. 2, pp. 343-365, Sep. 1992. http://dx.doi.org/10.1007/BF01208929

[12] M. Ali and L. M. Saha, "Local Lyapunov exponents and characteristics of fixed/periodic points embedded within a chaotic attractor," J. Zhejiang Univ.-SCI A, vol. 6, pp. 296-304, Apr. 2005.

[13] M. Hènon, "A two-dimensional mapping with a strange attractor," Commun. Math. Phys., vol. 50, pp. 69-77, Feb. 1976. 
Int'I Journal of Computing, Communications \& Instrumentation Engg. (IJCCIE) Vol. 3, Issue 2 (2016) ISSN 2349-1469 EISSN 2349-1477

http://dx.doi.org/10.1007/BF01608556

[14] H. Kawakami and K. Kobayashi, "Computer experiments on chaotic solutions of $x(t+2)-a x(t+1)-x^{2}(t)=b$," Bulletin of Faculty of Engineering, The University of Tokushima, vol. 16, pp. 29-46, Apr. 1979. 